\title{
Measuring access to emergency obstetric care in rural Zambia
}

\author{
Adam C. Levine • Regan H. Marsh • Sara W. Nelson • \\ Lynda Tyer-Viola $\cdot$ Thomas F. Burke
}

Received: 26 March 2008 / Accepted: 29 April 2008 / Published online: 31 May 2008

(C) The Author(s) 2008

\begin{abstract}
Background Global health experts identify emergency obstetric care (EmOC) as the most important intervention to improve maternal survival in low- and middle-income countries. In Zambia, 1 in 27 women will die of maternal causes, yet the level of availability of EmOC is not known at the provincial level.

Aims Our goal was to develop a tool to measure the availability of EmOC in rural Zambia in order to estimate pregnant women's access to this life-saving intervention.

Methods We created an instrument for determining the availability of EmOC based on the supplies and medicines in stock at health facilities as well as the skill level of health workers. We then surveyed a random sample of 35 health centres in the Central Province of Zambia using our novel instrument.

Results We graded health centres based on their ability to provide the six basic functions of EmOC: administering
\end{abstract}

Massachusetts Medical Society Medical Student and Resident Poster Symposium Poster: "Measuring Access to Emergency Obstetric Care in Rural Zambia" Waltham, Massachusetts, April 2007.

International Conference on Emergency Medicine (ICEM) Oral Presentation: "Measuring Access to Emergency Obstetric Care in Rural Zambia" San Francisco, California, April 2008.

Disclaimer The views expressed in this paper are those of the author (s) and not those of the editors, editorial board or publisher.

A. C. Levine $(\bowtie) \cdot$ R. H. Marsh $\cdot$ S. W. Nelson

Department of Emergency Medicine,

Brigham and Women's Hospital,

75 Francis Street,

Boston, MA 02115, USA

e-mail: alevine2@partners.org

L. Tyer-Viola $\cdot$ T. F. Burke

Center for Global Health, Massachusetts General Hospital,

Boston, MA, USA parenteral antibiotics, administering parenteral oxytocics, administering parenteral anticonvulsants, performing manual removal of the placenta, removing retained products of conception and performing assisted vaginal delivery. Of the 29 health centres providing delivery care, $65 \%$ (19) were graded as level 1 or 2, 28\% (8) as level 3 or 4 and $7 \%$ (2) as level 5. No health centre received a grade of level 6 .

Conclusion The availability of EmOC in the Central Province of Zambia is extremely limited; the majority of health centres provide only one or two basic functions of EmOC, and no health centres perform all six functions. Our grading system allows for inter- and intra-country comparisons by providing a systematic process for monitoring access to EmOC in rural, low-income countries similar to Zambia.

Keywords Emergency medical services .

Emergency obstetric care - Evaluation studies .

Rural health . World health

\section{Introduction}

The fifth Millennium Development Goal (MDG5) established the reduction of maternal mortality as a priority for the international community [1]. Sub-Saharan Africa accounts for over half of the estimated 536,000 global annual maternal deaths, and in some regions, the maternal mortality ratio (MMR) exceeds 900 per 100,000 live births $[2,3]$. Seventy per cent of deaths are attributed to haemorrhage, hypertensive diseases, sepsis/infection, unsafe abortion and obstructed labour [4]. Most maternal deaths occur during the third trimester of pregnancy and during the first week after delivery, with the first and second day after delivery representing the highest risk to the mother [5]. Given that the primary causes of maternal 
mortality are difficult to predict in advance and nearly impossible to prevent, global health experts, including the World Health Organization (WHO), UN Children's Fund (UNICEF) and UN Population Fund (UNFPA) have advocated for improved access to emergency obstetric care (EmOC) as the best means for reducing maternal mortality in low- and middle-income countries [6]. Maternal mortality represents one of the few global health crises for which access to high-quality emergency care represents the most cost-effective solution.

Despite the comparatively high frequency of maternal deaths in the poorest countries, individual deaths are still rare events, making measurement of maternal mortality challenging. Given this context, global health experts advocate the use of surrogate markers to measure the availability and quality of emergency obstetric health care services [6]. By measuring improvements in these surrogate markers over time, governments can track progress towards their overall goal of reducing maternal mortality. In 1997, WHO, UNICEF and UNFPA published Guidelines for Monitoring the Availability and Use of Obstetric Services (UN Guidelines); these guidelines define specific process indicators to measure the "minimum acceptable" level of access to EmOC in any given region [6]. The UN Guidelines divide health facilities into two groups, basic EmOC facilities and comprehensive EmOC facilities, based on their ability to perform defined signal functions. Basic EmOC facilities can:

1. Administer parenteral antibiotics

2. Administer parenteral oxytocics

3. Administer parenteral anticonvulsants

4. Perform manual removal of placenta

5. Perform removal of retained products

6. Perform assisted vaginal delivery

Comprehensive EmOC facilities can perform all six basic functions plus Caesarean section and blood transfusion. The UN Guidelines recommend at least four basic EmOC facilities and one comprehensive EmOC facility for every 500,000 people in the population [6].

While the UN process indicators have been used successfully in a variety of settings, some limitations have been identified [7-11]. To qualify as an EmOC facility, a health centre or hospital must have performed each of the six signal functions at least once within the past 3 months. However, some facilities do not see enough complicated deliveries to perform these functions on a regular basis, and thus are categorized as "non-EmOC". This limitation may underestimate the contribution of these facilities to local health care [12].

In Zambia, the current lifetime risk of a woman dying due to pregnancy is 1 in 27 [2]. The Central Province in Zambia is the site of a collaborative initiative between the
Government of Zambia and the Massachusetts General Hospital Center for Global Health to reduce maternal mortality by improving access to EmOC. The Central Province, one of the poorest and most rural regions of the country, was designated by the Government of Zambia as a pilot region for the initiative. Before launching the initiative, we were asked to perform a baseline assessment of the current availability of basic EmOC in the Central Province of Zambia, as no previous study had investigated the capacity of the Central Province health care system to meet existing standards for basic EmOC.

The goals of our study were twofold. First, we had to develop a novel tool for measuring the availability of basic EmOC in a rural area with a low rate of deliveries where the established UN process indicators would be likely to underestimate access to EmOC. Second, by surveying a representative sample of health facilities, we sought to use our novel tool to determine the current availability of EmOC in the Central Province of Zambia.

\section{Methods}

Study design/setting

We conducted a baseline assessment of EmOC capacity at government health centres in the Central Province of Zambia. In rural Zambia, health centres provide almost all aspects of health care, including management of emergency conditions. True emergency departments are not available. To create a representative sample of health centres, we used an updated registry of all government health centres provided by the Ministry of Health [13]. We randomly selected $30 \%$, or 35 , of the 110 health centres within the Central Province for our baseline assessment, as recommended by UN guidelines for measuring access to EmOC [6]. Randomization was achieved using the SPSS 14.0 random sample function.

Our study was approved by the National and Central Province Ministry of Health, as well as by the Brigham and Women's Hospital/Massachusetts General Hospital Institutional Review Board.

Methods of measurement

We created novel survey instruments based on international guidelines for developing EmOC programmes in lowincome countries and prior studies of maternal and infant health conducted by government and non-government organizations in Zambia. Our surveys were reviewed by both officials in the national Ministry of Health and practicing midwives and gynaecologists for content and language. 
In order to identify whether health facilities had the necessary equipment and staff to perform basic EmOC, we created two separate surveys. The first survey measured various aspects of health centre infrastructure, including the distance from the nearest hospital, the availability of electricity and clean water and the availability of medicines and supplies important to antenatal and delivery care. We administered this survey to the highest ranking staff member available on the day of our visit. The second survey focused on staff knowledge, skills and attitudes, including prior training, experience and capabilities. This survey was administered to all staff members performing deliveries at the health centre who were available on the day of our visit.

Our survey instruments were designed to identify the percentage of health centres in the Central Province providing basic EmOC. Though we defined basic EmOC using the same six signal functions recommended by the UN Guidelines, our instrument differed from those recommended by the UN in two ways. First, we defined a health centre as providing a signal function if it both had the equipment or medications necessary to perform the function in stock on the day of our visit and had at least one staff member with the skills necessary to perform the function working on the day of our visit. Second, instead of using a binary definition for health facilities (providing basic EmOC or not providing basic EmOC), we graded each health facility on a scale from 0 to 6 based on the number of signal functions that they could perform. Specifically, each health centre was given one point for the ability to perform each of the following:

\section{Administer parenteral antibiotics:}

At least one staff member felt comfortable giving IV medications and treating infection and the facility had at least one parenteral antibiotic in stock on the day of our visit.

2. Administer parenteral oxytocics:

At least one staff member felt comfortable giving IV medications and treating haemorrhage and the facility had oxytocin in stock on the day of our visit.

3. Administer parenteral anticonvulsants:

At least one staff member felt comfortable giving IV medications and treating high blood pressure or seizures and the facility had magnesium sulphate in stock on the day of our visit.

4. Perform manual removal of placenta:

At least one staff member felt comfortable performing manual removal of the placenta.

5. Perform removal of retained products:

At least one staff member felt comfortable removing retained products of conception and the facility had a working aspiration syringe or curette on the day of our visit.

6. Perform assisted vaginal delivery:

At least one staff member felt comfortable performing assisted vaginal delivery and the facility had a working vacuum extractor or vaginal forceps on the day of our visit.

Finally, we obtained census data from the Zambia Central Statistics Office on population and average growth rate for each health centre catchment area.

Data collection and processing

From November 2006 to February 2007, study personnel visited the randomly selected health centres within the Central Province. Study personnel administered the infrastructure/ medication/supplies survey to the most senior staff member available on the day of our visit. Equipment was recorded as available if it was clearly operational on the day of our visit. Similarly, medications or supplies that were out of stock on the day of their visit were recorded as not available, in order to present a realistic picture of what women visiting the health centre on an average day could expect.

After administering the first survey, study personnel administered the staff survey to all available staff members at the health centre who reported performing any deliveries in the past year. In Zambia, the vast majority of professionally attended deliveries are performed by either nurses, who complete 2-3 years of post-secondary school training including basic obstetric care, or midwives, who have completed 3-4 years of post-secondary training with a focus in obstetrics. Occasionally, deliveries will be performed by a clinical officer, with 3 years of general clinical training, or another health professional without specific obstetric training. All surveys were administered in English, and all professionally trained staff at government health centres and hospitals spoke English fluently. Study personnel collected data by hand on survey sheets at the time of the interviews and later transcribed them into an electronic database.

\section{Primary data analysis}

We analysed our data using SPSS 14.0. Using Zambian census data, we derived expected delivery rates for each health centre catchment area, adjusted for 2006.

Descriptive statistics were used to analyse the data from our surveys, including infrastructure capacity, the availability of medications and supplies and staff knowledge and skills. We combined data from these surveys to create a novel measure of the availability of basic EmOC at each Central Province health centre.

\section{Results}

Study personnel visited 33 of the 35 selected health centres, a response rate of $94 \%$. One randomly selected health 
centre was located on an Air Force Base and required national security clearance and a second health centre was not accessible by motor vehicle due to heavy rains at the time of our survey.

\section{Demographic data}

The median population served by the randomly selected health centres in our study was 8,059, ranging from 1,050 to 25,879 people. Based on Zambia census data, the expected delivery rates for 2006 for each of the health centre catchment areas ranged from 49 to 1,082, with a median of 334. Several prior studies of maternal health in developing countries have found that an average of $15 \%$ of pregnancies will experience at least one complication [6, 14, 15]. Using this estimate, the median number of expected complications in each health centre catchment area during the 3 months prior to our survey would have been 12 , with a range of 2-38 complications per catchment area. Therefore, even if all complications had presented to health centres, a sizable proportion of the health centres in the central province would not have seen enough complications to even have the opportunity to perform the six signal functions of basic EmOC in the 3 months prior to our survey.

\section{Medication and supplies}

Table 1 lists the availability of medications, supplies and equipment necessary for the delivery of basic EmOC. In general, nearly all facilities had IM penicillin available, though few had IV ampicillin or gentamicin. About a third of facilities had oxytocin for treating post-partum haemorrhage, and a quarter of facilities had magnesium for treating eclampsia. Few facilities have the necessary equipment to perform removal of retained products of conception $(9 \%)$ or assisted vaginal delivery (15\%).

Table 1 Available EmOC medications and equipment at health centres $(\mathrm{HC})$

\begin{tabular}{lll}
\hline EmOC supplies & HC with supplies & HC counted \\
\hline Oxytocin & $36.4 \%(12)$ & 33 \\
Magnesium sulphate & $27.3 \%(9)$ & 33 \\
Ampicillin & $27.3 \%(9)$ & 33 \\
Penicillin & $96.2 \%(25)$ & $26^{\mathrm{a}}$ \\
Gentamicin & $51.5 \%(17)$ & 33 \\
Vacuum extractor & $6.1 \%(2)$ & 33 \\
Forceps & $9.1 \%(3)$ & 33 \\
Speculum & $54.5 \%(18)$ & 33 \\
Aspiration syringe/curette & $9.1 \%(3)$ & 33 \\
Blood transfusion & $3.0 \%(1)$ & 33
\end{tabular}

${ }^{a}$ Penicillin was added to the survey when it became clear that it was the primary parenteral antibiotic available at most health centres

\section{Staff data}

When asked about their comfort in performing specific skills related to delivery, $100 \%$ of health centre staff members reported comfort in performing simple vaginal delivery at health centres. The vast majority also reported feeling comfortable giving IV medications (89\%), treating infection $(100 \%)$, treating haemorrhage $(83 \%)$ and manually removing the placenta $(80 \%)$. About half felt comfortable treating high blood pressure or seizures, or removing retained products of conception. Few felt comfortable with assisted vaginal delivery $(6 \%)$ or performing a Caesarian section $(0 \%)$. Table 2 contains data on the comfort of staff members with specific procedures.

Access to emergency obstetric care

Each of the health centres was then graded on a scale from 1 to 6 based on the number of functions of basic EmOC that they could be expected to perform. Table 3 and Fig. 1 provide a summary of the level of basic EmOC available in the Central Province of Zambia using this novel method.

As can be seen from Table 3 and Fig. 1, none of the health centres in the Central Province have the capacity to perform basic EmOC, as defined by the UN Guidelines. Using our novel tool, of the 29 health centres providing delivery care, $65 \%$ [19] were graded as level 1 or $2,28 \%$ [8] as level 3 or 4 and 7\% [2] as level 5. No health centre received a grade of level 6 . While most have the capacity to give parenteral antibiotics and perform manual removal of the placenta, far fewer can provide parenteral oxytocics or anticonvulsants. Few facilities can perform removal of retained products of conception or assisted vaginal delivery.

\section{Discussion}

With the Millennium Development Goals, the international community established the reduction of maternal mortality

Table 2 Health centre staff's comfort performing EmOC skills

\begin{tabular}{lll}
\hline Skill & $\begin{array}{l}\text { Staff comfortable } \\
\text { with skill }\end{array}$ & $\begin{array}{l}\text { Staff } \\
\text { counted }\end{array}$ \\
\hline Vaginal delivery & $100 \%(35)$ & 35 \\
Assisted vaginal delivery & $5.7 \%(2)$ & 35 \\
Manual removal of placenta & $80.0 \%(28)$ & 35 \\
Removal of retained products & $40.0 \%(14)$ & 35 \\
Giving IV medications & $88.6 \%(31)$ & 35 \\
Treating infection & $100 \%(35)$ & 35 \\
Treating haemorrhage & $82.9 \%(29)$ & 35 \\
Treating high blood pressure & $51.4 \%(18)$ & 35 \\
$\quad$ or seizures & & \\
\hline
\end{tabular}


Table 3 Level of basic EmOC at health centres $(\mathrm{HC})$
$\%$ of $\mathrm{HC}$

Total HC counted

Administer parenteral antibiotics

Administer parenteral oxytocics

Administer parenteral anticonvulsants

Perform manual removal of placenta

Perform removal of retained products

Perform assisted vaginal delivery

Level of basic EMOC

Health centres not performing deliveries

Level 0

Level 1

Level 2

Level 3

Level 4

Level 5

Level 6

$\begin{array}{ll}89.7 \%(26) & 29 \\ 34.5 \%(10) & 29 \\ 17.2 \%(5) & 29 \\ 82.8 \%(24) & 29 \\ 6.9 \%(2) & 29 \\ 3.4 \%(1) & 29 \\ & \\ 12.1 \%(4) & 33 \\ 0 \% & 29 \\ 21.2 \%(7) & 29 \\ 36.4 \%(12) & 29 \\ 15.2 \%(5) & 29 \\ 9.1 \%(3) & 29 \\ 6.1 \%(2) & 29 \\ 0 \% & 29\end{array}$

as a high priority [1]. In Zambia, the MMR is estimated at 830 per 100,000 live births [2]. However, even in areas with high maternal mortality, it is difficult to evaluate changes in maternal mortality due to the rarity of individual deaths [16]. The 1997 UN Guidelines established both the "minimum acceptable" package for obstetric care and a methodology for measuring process indicators, as surrogate markers of maternal mortality. In our study we developed a novel tool to assess access to EmOC in the Central Province of Zambia, an area selected by the government of Zambia for a new programme to reduce maternal and infant deaths.

Previous studies have found that $34 \%$ of women in this region deliver with a skilled attendant and less than $2 \%$ are by Caesarean section [17]. Variable quality of care, even in supervised deliveries, has been identified as an area of concern and a potential barrier to adequate EmOC [18]. With infrequent institutional deliveries, it can be difficult

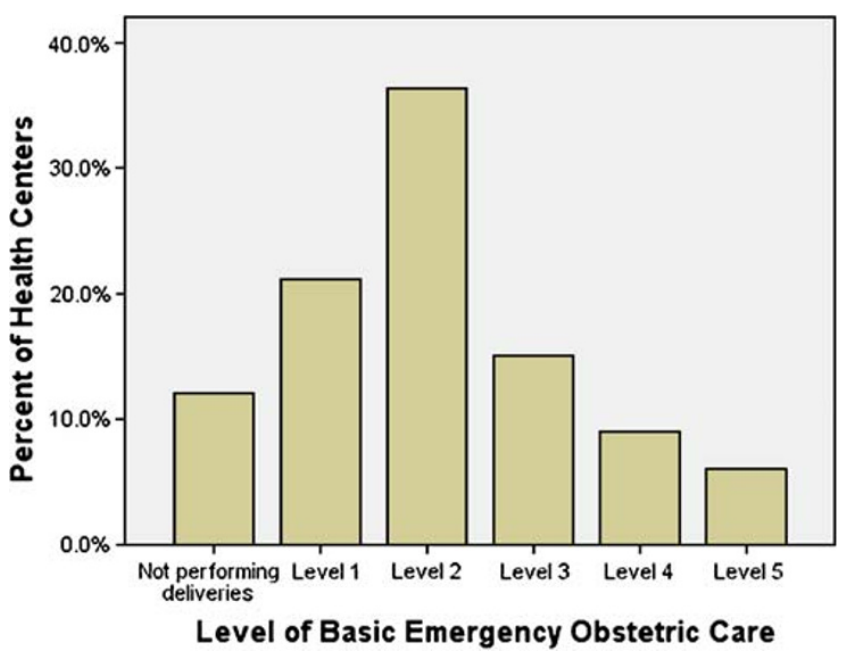

Fig. 1 Level of basic EmOC at health centres for trained providers to maintain their skills. While all staff felt comfortable performing spontaneous vaginal deliveries, many identified limitations, particularly with administering parenteral oxytocics, performing assisted vaginal delivery and removing retained products of conception. Though the number of obstetric complications at any given health centre is low, the staff in these centres feel limited in their ability to identify and manage these life-threatening complications.

In addition to limitations in staff capacity, we found notable gaps in medication supplies and equipment. Oxytocics and magnesium sulphate were not available in the majority of heath centres, and antibiotic options were inadequate. Less than $15 \%$ of health centres had the equipment necessary to perform assisted vaginal delivery.

Previous studies have identified limitations of the UN process indicators, which categorize health facilities as providing basic EmOC if they have performed each of the six signal functions at least once within the previous 3 months [19-23]. First, the process indicators cannot assess quality of care, such as whether the obstetric complications are being appropriately managed [22]. While our tool does not address quality, it does account for the individual provider's self-reported capacity to perform a skill. Second, some facilities do not have enough complicated deliveries to perform all six signal functions within a 3-month time frame and are thus classified as "nonEmOC"; for example, health centres rarely perform assisted vaginal delivery $[22,23]$. The Averting Maternal Death and Disability Program advocates using a designation of "basic minus one" for these facilities, to accurately reflect their contribution [23]. However, this system is unable to assess the capacity of facilities where all complications occur on an infrequent basis.

Using the established UN process indicators for basic EmOC, all of the health centres in our sample would be 
considered "non-EmOC"; none had performed all six of the basic functions within the previous 3 months. Indeed, for many it would have been impossible to perform all six signal functions, given the low number of expected obstetric complications in their catchment area. Given this context, our novel tool provides a method to assess capacity of individual health centres to provide EmOC, by assessing the providers' self-reported ability to perform the individual skills plus the presence of necessary supplies on the day of our survey. In our study, $35 \%$ of the selected health centres were providing three or more of the UN signal functions. Our tool recognizes these health centres' contribution to local health care. Use of a grading system also allows for recognition of improvements in capacity. Our tool delineates differences between those facilities that truly provide minimal services (grade 0-2) and those that have more extensive capabilities (grade 3-6). As providers are trained, and improvements are made in equipment and supplies, governments and other monitors can track health centre improvement.

\section{Limitations}

In this study, we evaluated the presence or absence of medications and supplies at the time of our visit and not the quality or quantity of supplies. Clearly, the total stock of a medicine and the quality of delivery equipment may have bearing on the delivery of care. In addition, the staff surveys were done with all the available providers that reported delivering babies in the past year. It is possible that the most experienced midwife or nurse was not present on the day of our visit. Our grading system was based on the level of EmOC available on the day of our visit - a measure that we feel best represents the experience of the average woman who presents for delivery. Our assessment of health centre staff's skills was based on their self-reported personal comfort in performing specific skills related to delivery and was not based on an objective measure of competence. At health centres with few deliveries, such skills could be assessed by a well-developed simulation or by observing staff in a busier hospital. Neither of these options was possible during our study. Finally, the study personnel were only able to visit 33 of the 35 randomly selected health centres, a response rate of $94 \%$.

\section{Conclusion}

Emergency obstetric care in the Central Province of Zambia is limited. This study provides data for programmatic use to improve the capacity of the local health care system to care for pregnant women and infants. Additionally, our tool can be used to continually assess the individual health facilities and their ability to meet UN standards for EmOC. A focus on continued education of skilled providers and on increased delivery of emergency obstetric services remains the key to future reductions in maternal morbidity and mortality.

\section{Conflicts of interest None.}

Funding Funding for this study was provided by the Center for Global Health at Massachusetts General Hospital.

Open Access This article is distributed under the terms of the Creative Commons Attribution Noncommercial License which permits any noncommercial use, distribution, and reproduction in any medium, provided the original author(s) and source are credited.

\section{References}

1. United Nations. United Nations Millennium Declaration. Resolution adapted by the General Assembly, 55th Session of the United Nations General Assembly, New York, 18 September 2000

2. WHO, UNICEF, UNFPA, the World Bank (2007) Maternal Mortality in 2005; estimates developed by WHO, UNICEF, UNFPA, and The World Bank. World Health Organization, Geneva

3. Hill K, Thomas K, AbouZahr C, Walker N, Say L, Inoue M, Suzuki E, Maternal Mortality Working Group (2007) Estimates of maternal mortality worldwide between 1990 and 2005: an assessment of available data. Lancet 370:1311-1319

4. Khan KS, Wojdyla D, Say L, Gülmezoglu AM, Van Look PFA (2006) WHO analysis of causes of maternal death: a systematic review. Lancet 367:1066-1074

5. Ronsmans C, Graham WJ et al (2006) Maternal mortality: who, when, where, and why. Lancet 368:1189-1200

6. WHO/UNICEF/UNFPA (1997) Guidelines for monitoring the availability and use of obstetric services. United Nations Children's Fund, New York

7. Urassa DP, Carlstedt A, Nyström L, Massawe SN, Lindmark G (2005) Are process indicators adequate to assess essential obstetric care at district level? - a case study from Rufiji district, Tanzania. Afr J Reprod Health 9(3):100-111

8. Bullough C, Meda N, Makowiecka K, Ronsmans C, Achadi EL, Hussein J (2005) Current strategies for the reduction of maternal mortality. BJOG 112:1180-1188

9. Averting Maternal Death and Disability Working Group on Indicators (2002) Averting maternal death and disability. Program note. Using UN process indicators to assess needs in emergency obstetric services: Pakistan, Peru and Vietnam. Int J Gynaecol Obstet 78:275-282

10. Kayongo M, Rubardt M, Butera J, Abdullah M, Mboninyibuka D, Madili M (2006) Making EmOC a reality-CARE's experiences in areas of high maternal mortality in Africa. Int $\mathrm{J}$ Gynaecol Obstet 92:308-319

11. Chowdhury ME, Botlero R, Koblinsky M, Saha SK, Dieltiens G, Ronsmans C (2007) Determinants of reduction in maternal mortality in Matlab, Bangladesh: a 30-year cohort study. Lancet 370:1320-1328 
12. Paxton A, Bailey P, Lobis S (2006) The United Nations Process Indicators for emergency obstetric care: reflections based on a decade of experience. Int J Gynaecol Obstet 95:192-208

13. Health Institutions in Zambia (2002) A listing of health facilities according to levels and location for 2002. Central Board of Health, Zambia

14. Hartfield VJ (1980) Maternal mortality in Nigeria compared with earlier international experience. Int J Gynaecol Obstet 18:70-75

15. Hibbard LT (1978) Complications of pregnancy and delivery. In: Benson RC (ed) Current obstetric and gynecological diagnosis and treatment. Lange, Los Altos, pp 664-686

16. Paxton A, Maine D, Freedman L, Fry D, Lobis S (2005) The evidence for emergency obstetric care. Int J Gynaecol Obstet 88:181-193

17. Central Statistical Office (Zambia), Central Board of Health (Zambia), ORC Macro (2003) Zambia Demographic and Health Survey 2001-2002. Central Statistical Office, Central Board of Health, and ORC, Calverton, MD

18. Koblinsky M, Matthews Z, Hussein J et al (2006) Going to scale with professional skilled care. Lancet 368:1377-1386

19. Ronsmans C, Campbell OMR, McDermott J, Koblinsky M (2002) Questioning the indicators of need for obstetric care. Bull World Health Organ 80(4):317-324

20. Ross JA, Begala JE (2005) Measures of strength for maternal health programs in 55 developing countries: the MNPI study. Matern Child Health J 9(1):59-70

21. Goodburn EA (2002) Using process indicators to monitor and evaluate obstetric services in developing countries. J Am Med Womens Assoc 57(3):145-148

22. Fauveau V, Donnay F (2006) Can the process indicators for emergency obstetric care assess the progress of maternal mortality reduction programs? An examination of UNFPA Projects 2000 2004. Int J Gynaecol Obstet 93:308-316

23. Paxton A, Bailey P, Lobis S (2006) The United Nations Process Indicators for emergency obstetric care: reflections based on a decade of experience. Int J Gynaecol Obstet 95:192-208

Adam C. Levine received his medical degree from the University of California, San Francisco, and his MPH degree from the University of California, Berkeley with a concentration in global health. He is a senior resident at the Brigham and Women's Hospital/Massachusetts General Hospital Harvard Affiliated Emergency Medicine Residency. He currently serves as the Chair of the Emergency Medicine Resident's Association (EMRA) International Emergency Medicine Committee and as Editor of the annual EMRA International
Emergency Medicine Literature Review. His research interests include improving the delivery of emergency care and humanitarian relief in the developing world.

Regan H. Marsh received her medical degree in 2004 from the University of Pennsylvania in Philadelphia. She is currently chief resident at the Harvard Affiliated Emergency Medicine Residency at Brigham and Women's and Massachusetts General Hospitals in Boston. Her research interests include maternal and infant health and leadership development.

Sara W. Nelson received her medical degree in 2005 from Harvard Medical School. She is currently a resident in Emergency Medicine at the Harvard Affiliated Emergency Medicine residency in Boston, where next year she will serve as chief resident. Her research interests include resident education and maternal and infant health.

Lynda Tyer-Viola received her $\mathrm{PhD}$ in Nursing Science from Boston College and completed her post doctoral fellowship in HIV and pregnancy research at Massachusetts General Hospital (MGH), Boston, Massachusetts. She is currently a Nurse Scientist in the MGH Yvonne Munn Nursing Research Institute, an assistant professor at the MGH Institute for Health Professions in Boston, Massachusetts, and a Senior Advisor for the MGH Center for Global Health for the Maternal Infant Health Initiative: Zambia.

Thomas F. Burke, MD, FACEP, is the director of the Massachusetts General Hospital (MGH) Center for Global Health and is also a senior emergency physician at MGH and Children's Hospital-Boston. Prior to joining the MGH, Dr. Burke was Associate Clinical Director of the Department of Emergency Medicine at Brigham and Women's Hospital and a faculty member for the hospital's Division of International Health and Humanitarian Programs. He also was Director of Development for the Harvard Humanitarian Initiative at the Harvard School of Public Health. 